\title{
Systemically Administered, Target Organ-Specific Therapies for Regenerative Medicine
}

\author{
Tero A. H. Järvinen ${ }^{1,2, *}$, Ulrike May ${ }^{1}$ and Stuart Prince ${ }^{1}$ \\ 1 School of Medicine, University of Tampere, 33520 Tampere, Finland; \\ E-Mails: ulrike.may@uta.fi (U.M.); stuart.prince@uta.fi (S.P.) \\ 2 Department of Orthopedics \& Traumatology, Tampere University Hospital, \\ 33520 Tampere, Finland \\ * Author to whom correspondence should be addressed; E-Mail: blteja@uta.fi; \\ Tel.: +358-44-285-4620.
}

Academic Editor: Francesc Cebrià

Received: 31 July 2015 / Accepted: 22 September 2015 / Published: 30 September 2015

\begin{abstract}
Growth factors and other agents that could potentially enhance tissue regeneration have been identified, but their therapeutic value in clinical medicine has been limited for reasons such as difficulty to maintain bioactivity of locally applied therapeutics in the protease-rich environment of regenerating tissues. Although human diseases are treated with systemically administered drugs in general, all current efforts aimed at enhancing tissue repair with biological drugs have been based on their local application. The systemic administration of growth factors has been ruled out due to concerns about their safety. These concerns are warranted. In addition, only a small proportion of systemically administered drugs reach their intended target. Selective delivery of the drug to the target tissue and use of functional protein domains capable of penetrating cells and tissues could alleviate these problems in certain circumstances. We will present in this review a novel approach utilizing unique molecular fingerprints ("Zip/postal codes") in the vasculature of regenerating tissues that allows target organ-specific delivery of systemically administered therapeutic molecules by affinity-based physical targeting (using peptides or antibodies as an "address tag") to injured tissues undergoing repair. The desired outcome of targeted therapies is increased local accumulation and lower systemic concentration of the therapeutic payload. We believe that the physical targeting of systemically administered therapeutic molecules could be rapidly adapted in the field of regenerative medicine.
\end{abstract}


Keywords: angiogenesis; tissue regeneration; in vivo phage display; decorin; vascular ZIP codes; regenerative medicine

\section{Local vs. Systemic Drug Delivery in Regenerative Medicine}

Adult tissues respond to injury differently. Some tissues, such as the bone, repair injuries with tissue that is identical to the original tissue. However, most tissues respond by undergoing a repair process that only partially restores the original tissue with the rest replaced by non-functioning, fibrotic scar tissue [1,2].

Numerous growth factors and other agents that could potentially enhance tissue regeneration have been identified, but their therapeutic application has been rather limited in clinical medicine $[1,3,4]$. There are several reasons for their limited use: it is difficult to maintain bioactivity of locally applied therapeutic agents in regenerating tissue because of lack of retention of the agent, poor tissue penetration, and instability of protein therapeutics in the protease-rich environment of the injured tissue $[4,5]$. Moreover, most injuries are not accessible with topical application of therapeutic molecules and multiple sites (tissues) of injury further limit the usefulness of local treatment.

Strikingly, all current efforts aimed at enhancing tissue repair with biologic drugs have been based on local application of therapeutic molecules to the injured site $[5,6]$. Although human diseases are treated with systemically administered drugs in general, systemic administration of growth factors has been ruled out due to concerns about their systemic use and potential safety. These concerns are warranted because the major problems in systemic drug therapy are that only a small proportion of administered drug reaches its intended target site(s). In addition, large molecules such as antibodies are poor at penetrating tissues and do not always reach the actual target cells [7-10]. Selective delivery of the drug to the target tissue and use of functional protein domains, such as cell penetrating peptides, capable of penetrating cells and tissues could alleviate some of these problems [10-13].

\section{Vascular Heterogeneity_-ZZip Codes" in Vasculature}

Our increased understanding of the structure of blood vessels on the molecular level has revealed a practical possibility for organ-specific therapeutic treatment of various human diseases with systemically administered drugs $[9,14]$. Recent research has shown that each organ has unique molecular structures in its blood vessels ("vascular ZIP codes") [9,14-17] (Figure 1). Each organ confers endothelial cells (ECs) in it with their "organotypic", i.e., unique tissue-specific, features $[9,14,15,18]$. Clusters of transcription factors, angiocrine growth factors, adhesion molecules and chemokines are expressed in unique combinations by the ECs of each organ and the blood vessels in each organ can be defined [15]. Furthermore, various diseases display disease-specific signatures on the vasculature of the diseased tissue [19]. This is especially evident for diseases such as different cancers, or tissue injury, which induce new blood vessels by angiogenesis [11]. The angiogenic blood vessels, in turn, are structurally distinct from the pre-existing blood vessels in the body [11,20]; a fact also highlighted by different types of stem cells being recruited to take part in the process [21]. 

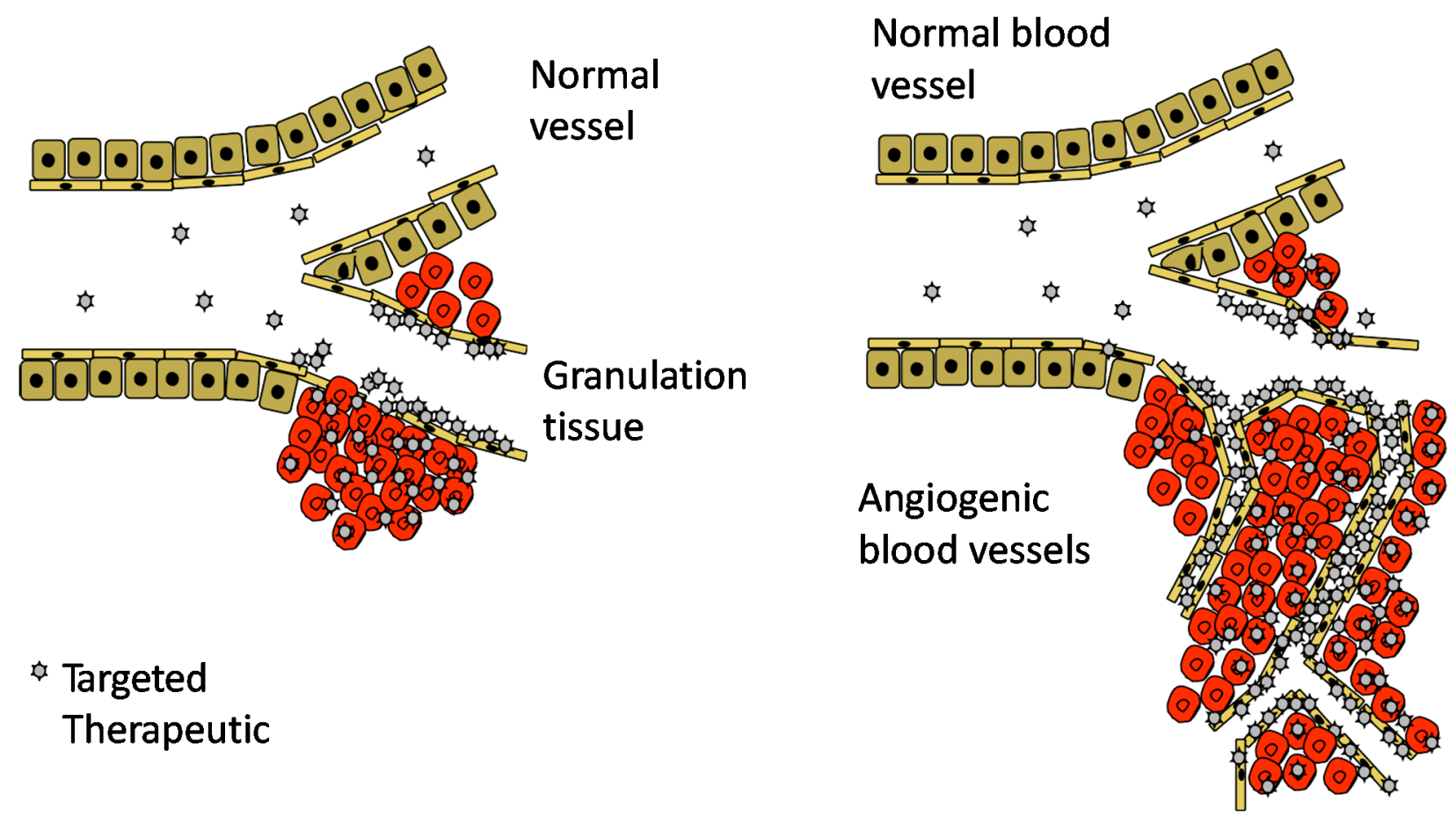

Figure 1. Target-specific drug therapies in tissue regeneration. Molecular fingerprints ("Zip/postal codes") in the angiogenic vasculature of the regenerating tissues allow target organ-specific delivery of the systemically administered therapeutic molecules by affinity-based physical targeting (using peptides or antibodies as an "address tag") to injured tissues undergoing repair. The desired outcome of the targeted therapies is similar to topical application: increased local accumulation and lower systemic concentration of the therapeutic payload.

The disease and organ-specific molecular "Zip codes" in blood vessels can be utilized for target organ-specific delivery of therapeutic molecules by affinity ligands [9,11,14,19]. Affinity-based physical targeting (synaphic, pathotrophic, or active targeting) makes use of these vascular ZIP codes, i.e., molecular markers that are specifically expressed at the target, and not elsewhere in the body, to accomplish selective targeting of systemically administered drugs to the target organ [11]. The desired outcome of the synaphic targeting is increased local accumulation and lower systemic concentration of the therapeutic payload [11].

\section{In Vivo Phage Display}

Vascular "ZIP codes" can be easily probed by in vivo phage display, a method first reported by Erkki Ruoslahti's group in 1996 [14]. In vivo phage display allows unbiased exploration of vascular diversity by random peptide libraries expressed in bacteriophage [22] (Figure 2). Phage display is a powerful method for peptide library screening that provides a physical linkage between peptides (i.e., the phenotype), which are displayed on the surface of a bacteriophage particle, and the encoding DNA (genotype) [22,23]. 


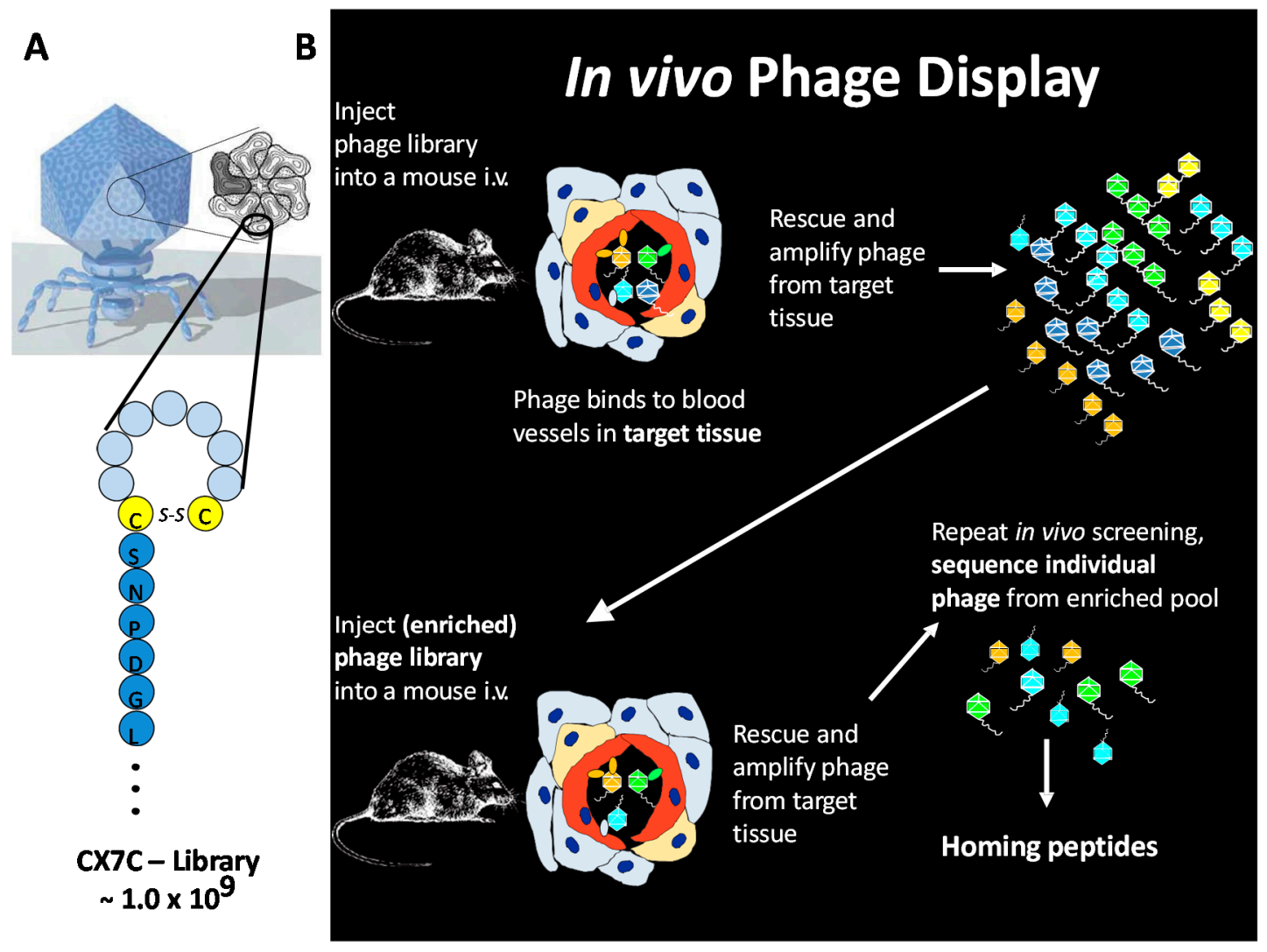

Figure 2. Schematic presentation of the principle of in vivo phage display. (A) A cyclic CX7C-peptide library is cloned onto the C-terminus of phage coat protein and 415 copies expressed per T7 phage via Select 415-1b; and (B) the phage library is injected into the circulation. As the homing peptides on the phage bind to endothelium in the tissues, there is an enrichment of phage that binds to the endothelium of the target tissue. Target tissue is homogenized, cell suspensions prepared, and the bound phage rescued and amplified by adding E. coli. The amplified phage pool recovered from the target tissue is re-injected into mice at a similar disease stage, and the screening cycle repeated several times to ensure phage clones that specifically bind (i.e., home) towards target are recovered. A set of phage clones is collected from the homing phage population that shows enrichment towards the target tissue. The peptide-encoding DNA inserts are amplified by PCR, and the PCR products sequenced. Modified from Ruoslahti [19].

Bacteriophage can be genetically modified to incorporate random protein sequences as fusions with the coat proteins at a diversity of billions of variants per library, close to the total number of possible permutations of a random amino acid sequence [22]. The outcome of generating a random phage library is a pool of billions of bacteriophages all identical to each other except for the protein motif expressed at the end of its coat protein. For in vivo selection, a library of phage displaying random peptides is injected systemically into the animals, followed by removal of target organ, amplification of the bound phage from the target organ, and subjecting the amplified pool to another round of selection in new animals [22]. In vivo peptide phage screening combines subtractive elements (removal of phage displaying pan-specific peptides by all other organs of the body except the given target organ) with positive selection at the target tissue [22]. In vivo phage display offers a unique opportunity to screen 
for potentially billions of protein-based drug candidates simultaneously in an in vivo setting [22]. Combination of in vivo phage display and bacterial 2-hybridization allows simultaneous identification of ligand-receptor pairs for target organ-specific targeting [24].

So far in vivo phage display has been used for several purposes in the field of regenerative medicine: to identify peptides capable of homing to the angiogenic blood vessels forming at injured tissues [20] and for the identification and illumination of neural structures during surgery [25].

\section{Angiogenesis-An Opportunity for Vascular Targeting in Regenerative Medicine}

Angiogenesis is a vital requirement for wound healing because the formation of new blood vessels allows a variety of mediators, nutrients, and oxygen to reach the healing tissue $[1-3,26]$. Angiogenesis is driven by tissue hypoxia, and inflammation, as both surviving cells and the inflammatory cells that have invaded the injured area secrete a large number of angiogenic growth factors [1,3,26,27]. During regeneration of injured tissue, angiogenic capillary sprouts invade the fibrin/fibronectin-rich clot and within a few days organize into a thick microvascular network throughout the granulation tissue [1-3,26,28]. The name, granulation tissue, actually derives from the granular appearance of abundant, newly formed blood vessels. As these newly formed blood vessels essentially fill up the granulation tissue forming at the site of injury and they differ in molecular structure from pre-existing vasculature, they provide an opportunity for vascular targeting of systemically administered drugs for purposes of enhancing the tissue regeneration process [20,29,30].

\section{CRK and CAR Vascular Homing Peptides for Regenerative Medicine}

We have reasoned that it could be possible to deliver systemically administered drugs into regenerating tissues in a target organ-specific fashion independent of the location of the injury in the body [20]. For that goal, we have probed injured tissues during angiogenesis with in vivo phage display to identify protein motifs capable of homing to the injured tissue after systemic administration [20]. We have identified two peptides that selectively target injured tissues: CARSKNKDC (CAR) and CRKDKC (CRK) [20]. CAR is homologous to heparin-binding sites in various proteins and shows the highest homology with the main heparin-binding site of the known angiogenic growth factor, bone morphogenetic protein 4 (BMP4). In line with that heparin binding site, CAR peptide binds to cell surface heparan sulfate proteoglycans (HSPGs) and utilizes HSPGs for efficient cell and tissue penetration [20], whereas the CRK peptide is not capable of cell and tissue penetration [20,31]. CRK peptide, in turn, has structural similarity to segments in thrombospondin type 1 and 3 repeats identified in a large number of proteins. These two vascular homing peptides have a distinct difference in their targeting ability; CAR peptide shows a preference for early stages of tissue regeneration, whereas CRK favors injured tissues at later stages of healing [20].

\section{Conjugated Delivery}

Conventional tissue-specific delivery has called for conjugation of a targeting element to the therapeutic molecule to obtain a targeted therapeutic molecule (Figure 3). The conjugation can be either done by chemical conjugation of the two functional domains or by expressing them together as a 
recombinant fusion protein. Prime examples of such recombinant fusion proteins include interleukin-10 (IL-10) fused together with the antibody scFv F8 (F8-IL10, i.e., Dekavil) that recognizes a domain of fibronectin expressed exclusively on inflammatory neovasculature [32-35]. This molecule is in clinical trials to treat arthritis [32-35]. Other examples include Angiopep, a peptide used to target nanoparticles loaded with therapeutic molecules to brains in e.g., Parkinson's disease [13] and the tumor homing NRG-peptide fused together with tumor necrosis factor $\alpha(\mathrm{TNF} \alpha)$ to induce anti-tumor and toxic reactions towards tumor cells [36,37].

The half-life of the most common targeting element, peptides, is usually short in circulation and their binding affinity for their respective receptor relatively low. Both of these features can be substantially improved by fusing the peptides to become part of a larger recombinant protein or by coating cells or nanoparticles with multiple copies of the peptide [11,38]. For example, we have obtained preliminary evidence that incorporation of CAR peptide to therapeutic proteins enhances CAR peptide's binding efficacy to its receptors to a nanomolar-level, and more so if the recombinant fusion protein is multimerized (expressed as a dimer). One-to-one peptide-payload conjugates can still be quite effective in targeting and avoiding side-effects in healthy tissues despite the large size difference between the peptide and the therapeutic domain, particularly when the peptide is used to augment an inherent affinity of the payload to the target [36,37,39]. A prime example of such complementary targeting is TNF $\alpha$ targeted to tumors with the NRG tumor-homing peptide; such a conjugate is now in phase 3 clinical trials [36,37], and CAR-peptide targeted decorin [39]. Decorin has been reported to home to angiogenic vasculature through a core protein-dependent interaction [40], but despite its inherent homing ability, the CAR peptide was able to enhance the accumulation of the fusion protein to sites of angiogenesis approximately 500\% over native decorin [39]. An illustrative example of using multiple copies of short targeting peptides is by "painting" the intended therapeutic domain with multiple copies of the peptide. Such an approach is utilized when targeting stem cells to desired loci by "painting" the stem cells with multiple copies of the homing peptide [38,41]. Both CAR and CRK peptides have been used successfully to target mesenchymal stem cells to infarcted myocardium after "painting" the cells with multiple copies of the peptide $[38,41]$. The benefit of using homing peptides that are also cell penetrating peptides (CPPs) is that such a peptide greatly increases the transport of the drug to the extravascular tissue [10,20,39] (Figure 3).

Even more remarkable is the fact that the targeting domain can enhance the activity of the therapeutic molecule [39]. We have found that a vascular homing peptide for a tissue or lesion often also binds to the corresponding parenchymal cells [11], and this is the case with CAR, which also recognizes the granulation tissue in wounds [39]. This localization of the peptide receptor drives the translocation of the blood vessel-bound peptide into the injured tissue, where the therapeutic payload can exert its functions more potently as it is anchored on the target cells. We were able to show that the anchorage to target cells provided by CAR peptide's fusion to decorin, enhanced decorin's biological potency to neutralize transforming growth factor- $\beta$ (TGF- $\beta$ ) [39]. Later, Hubbell et al. generated "super" growth factors, i.e., more potent growth factors than native ones, simply by fusing them with the heparin-binding domain of BMP2 (analogous to the CAR peptide, which is a close homologue to the heparin binding domain of BMP4) [6]. 
A

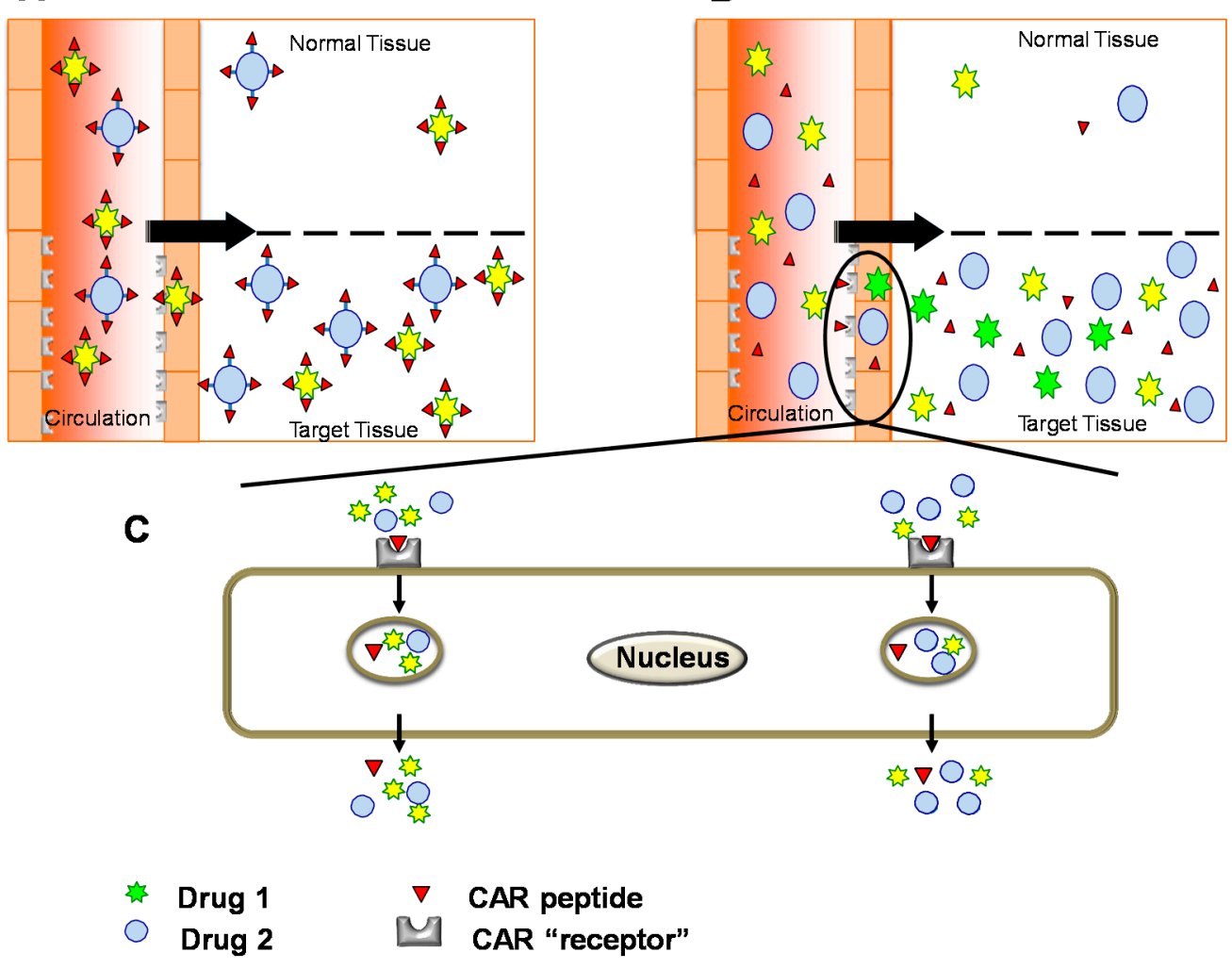

Figure 3. Systemically administered, targeted therapies: (A) The drugs are conjugated physically to the targeting device in conventional drug targeting. For protein-based therapeutics, targeting domain and therapeutic molecule are fused together as a recombinant protein with enhanced activity and tissue-specificity in conjugated delivery; (B,C) Bystander effect: Compounds co-injected with tissue-penetrating homing peptides are transported across the vessel wall and through tissue together with the peptides [12,42-45]. No physical conjugation is needed between the targeting peptide and drug; the cell penetrating homing peptide "sweeps" co-injected drugs to its target (homing) tissue in tissue-specific fashion $[12,42,45]$.

One of the benefits of using short peptides as targeting elements, is that the peptides are unlikely to be immunogenic in themselves because they are simply too small. For example, they are a fraction of the size of the highly variable complementary determining regions (CDRs) of therapeutic antibodies, which generally have an excellent drug safety and low immune reaction record [10]. Furthermore, when analyzing the homology of homing peptides (by BLAST), these short and stable cyclic peptides typically show high levels of homology to parts of native proteins found across a range of species indicating that the functional domain in the homing peptide is conserved between species (as is the case for both CRK and CAR, for example) [20]. This would predict high tolerance of the peptide by the immune system.

These pharmacological properties highlight the importance of advancing multi-functional, systemically administered, target-seeking recombinant fusion proteins. However, unless the target of a homing peptide is known to be unimportant for function of the immune system, cell proliferation, angiogenesis, etc., one cannot rule out the possibility of adverse effects caused by high doses of a homing peptide binding to its target receptor. Thus, it is necessary to elucidate the target and mechanism of homing peptides, and to investigate their safety. 


\section{Bystander Effect}

The latest results have revealed a tissue-penetrating transport pathway for certain targeting peptides that can be used to enhance drug targeting without requiring any conjugation between the therapeutic and targeting domains [43-45] (Figure 3). The essential features of the system called the "bystander effect" were first elucidated using a new RGD (an integrin binding sequence) peptide, termed iRGD because it internalizes into target cells [43,45] (Figures 3 and 4). Like conventional RGD peptides [46], iRGD accumulates at tumor vasculature as a result of integrin binding; but it is then cleaved by a protease to unmask a second binding motif, a so-called CendR motif (consensus: R/KXXR/K) [43-45]. The CendR motif binds to neuropilin-1, which activates a transport tissue-penetration and cell internalization pathway [43-45]. Remarkably, the CendR pathway is a bulk transport system. Once activated, it will sweep along any molecule or nanoparticle ("Bystander effect") that is present in the environment (Figure 3). Thus, it is not necessary to couple iRGD to the compound to be targeted; the therapeutic molecule can be simply co-injected with the vascular homing peptide capable of inducing the bystander effect, which will sweep the molecule into the target organ [42-45] (Figure 3).
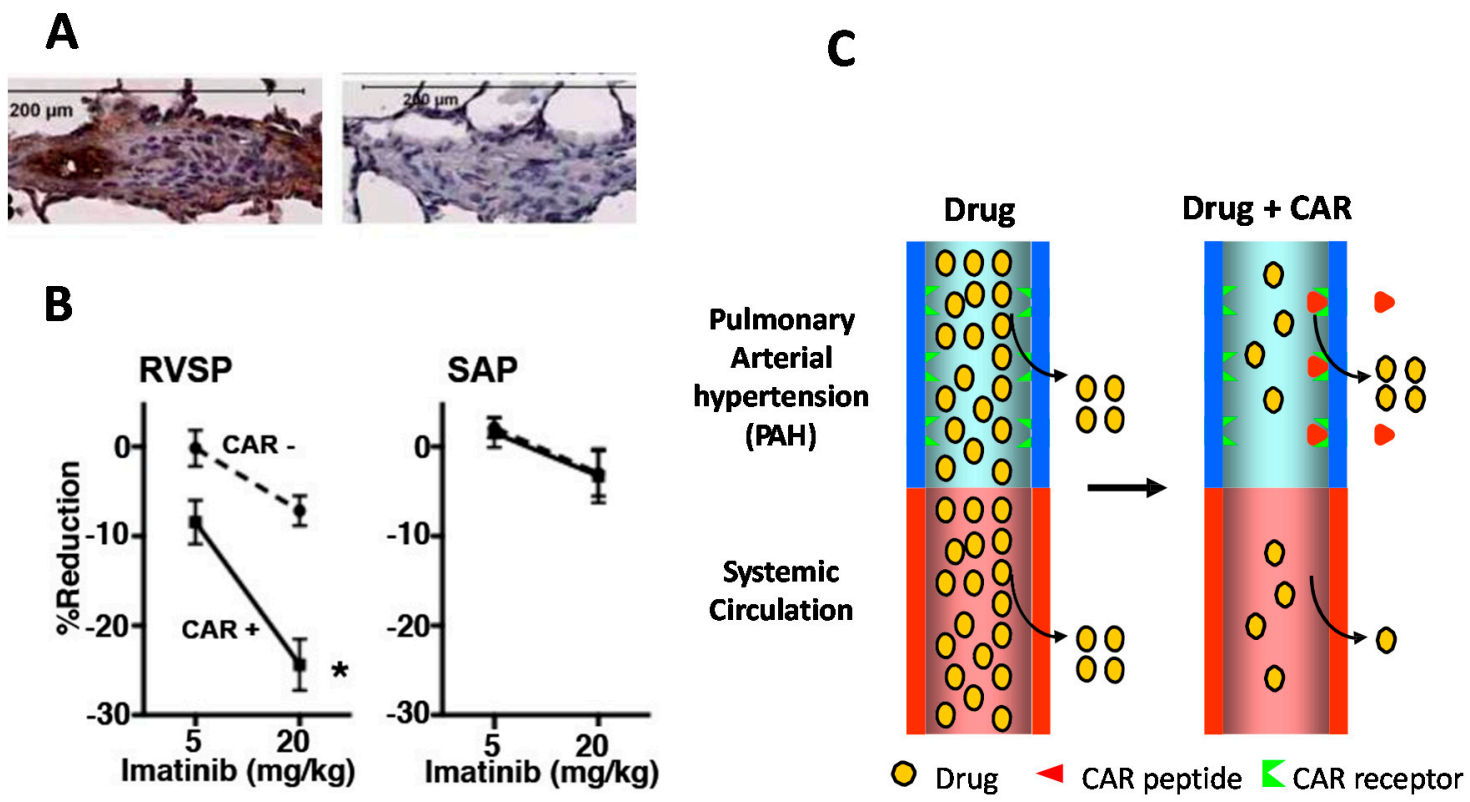

Figure 4. CAR peptide homes to inflammatory vasculature in pulmonary arterial hypertension (PAH) and induced target-tissue selective vasodilation in PAH. (A) PAH was induced in rats by a single subcutaneous injection of SU5416 and three week exposure to hypoxia $\left(10 \% \mathrm{O}_{2}\right)$ followed by six weeks of normoxia [42,47]. CAR peptide accumulates in remodeled pulmonary arteries (A/C: occlusive neointimal formation) [42,47]. Little signal was detected in lung lesions of the PAH rats administered with CAR mutant peptide; (B) CAR peptide was given orally (p.o.) at the same time with Imatinib (Gleevec). CAR peptide makes drugs tissue-selective in $\mathrm{PAH}$, i.e., the drugs are substantially more potent in the pulmonary vasculature (RVSP) where the PAH takes place, whereas the blood pressure lowering effect induced by CAR co-administration is unchanged on the systemic side of the circulation (SAP), * $p<0.05$; and (C) Schematic presentation of targeted delivery by the bystander effect induced by vascular homing peptide CAR. 
We have recently noted a bystander effect for a vascular homing and cell penetrating peptide that does not contain the CendR-motif [42] (Figures 3 and 4). We have shown that the CAR peptide, that does not have a CendR-sequence in it [42], is a very potent inducer of a bystander effect in pulmonary hypertension (PAH) [42] (Figure 4). Namely, CAR peptide homes to the inflammatory vasculature in a rat model of PAH and penetrates to inflammatory lung parenchyme [42,47] (Figure 4). We have shown that it can convert any vasodilator drug to become a target organ-specific vasodilator in that model of PAH; when a low dose of vasodilator is given together with CAR peptide, the drug accumulates in the diseased lungs and lowers the hypertension selectively only in the pulmonary circulation and leaves the systemic circulation unaffected for target organ-specific vasodilation [42] (Figure 4). It remains to be determined whether CAR- or CendR-peptides induce a bystander effect that could be used for therapeutic advantage in regenerative medicine.

\section{CAR-Decorin-Target Organ-Specific Systemically Administered Anti-Fibrotic Molecule}

Decorin (DCN) is the best characterized member of the extracellular small leucine-rich proteoglycan family. It is present in a variety of connective tissues, typically in association with or "decorating" collagen fibrils, and has substantial interest to clinical medicine owing to its well established anti-fibrotic and pro-regenerative effects [48-50]. DCN is a natural inhibitor of TGF- $\beta$ that shuts down the TGF- $\beta$ responses related to injury, cancer growth and inflammation [39,49-53]. In addition to blocking TGF- $\beta$, DCN also inhibits other important inducers of fibrosis/scarring; myostatin and connective tissue growth factor (CTGF/CCN2) [29,50,54-56]. The ability of decorin to prevent scar formation and fibrosis and to simultaneously promote tissue regeneration has been demonstrated in a large number of tissue injuries and diseases [49-51]. We have recently developed a systemically administered, targeted, e.g., inflammation and angiogenesis homing version of DCN core protein [39] (Figure 5). The angiogenesis and inflammation specificity of our enhanced DCN core protein is obtained by a fusion to the CAR homing peptide that functions as an address tag in the fusion protein and delivers systemically administered DCN to angiogenic and inflammatory vasculature [29,39] (Figures 5 and 6). CAR peptide can deliver increased amounts of DCN in a tissue-specific manner with a significant therapeutic advantage over ordinary DCN core protein in healing skin wounds [29,39] (Figures 5 and 6). Furthermore, the fusion of CAR to recombinant DCN enhances its neutralization effect on TGF- $\beta$ [39]. The molecular explanation for the enhanced biological activity of CAR-DCN is that the CAR peptide binds to HSPGs on the target cells. TGF- $\beta 1$, and TGF- $\beta 2$ in turn, also bind heparan sulfate and HSPG binding increases their biological activity [29,39] (Figure 5). Thus, CAR mediated binding of CAR-DCN to HSPGs may enhance the neutralizing effect of the fusion protein by bringing it into the proximity of the HSPG-binding TGF- $\beta$ s [29,39] (Figure 5). 
(1)

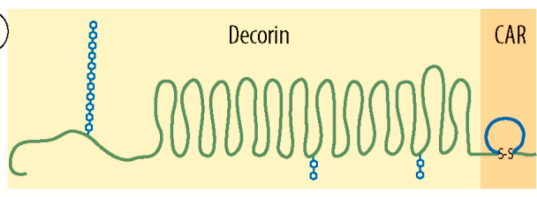

(2)

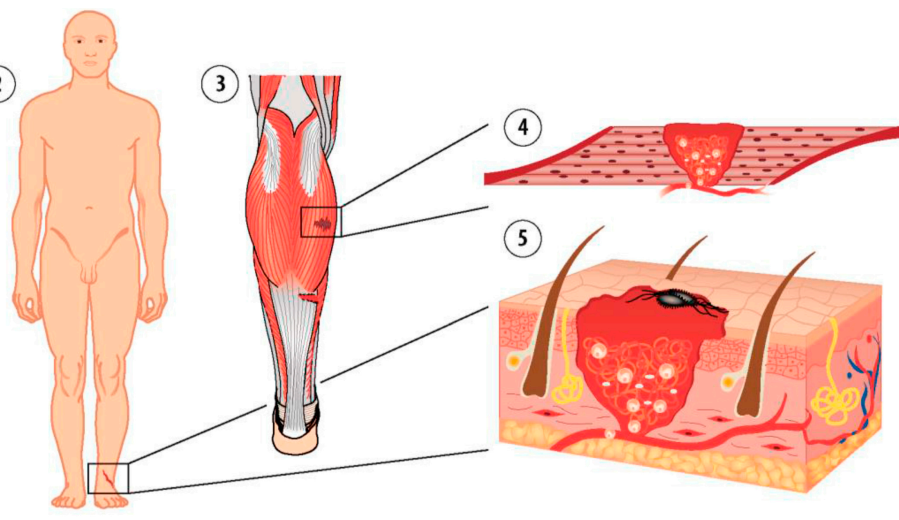

(6)

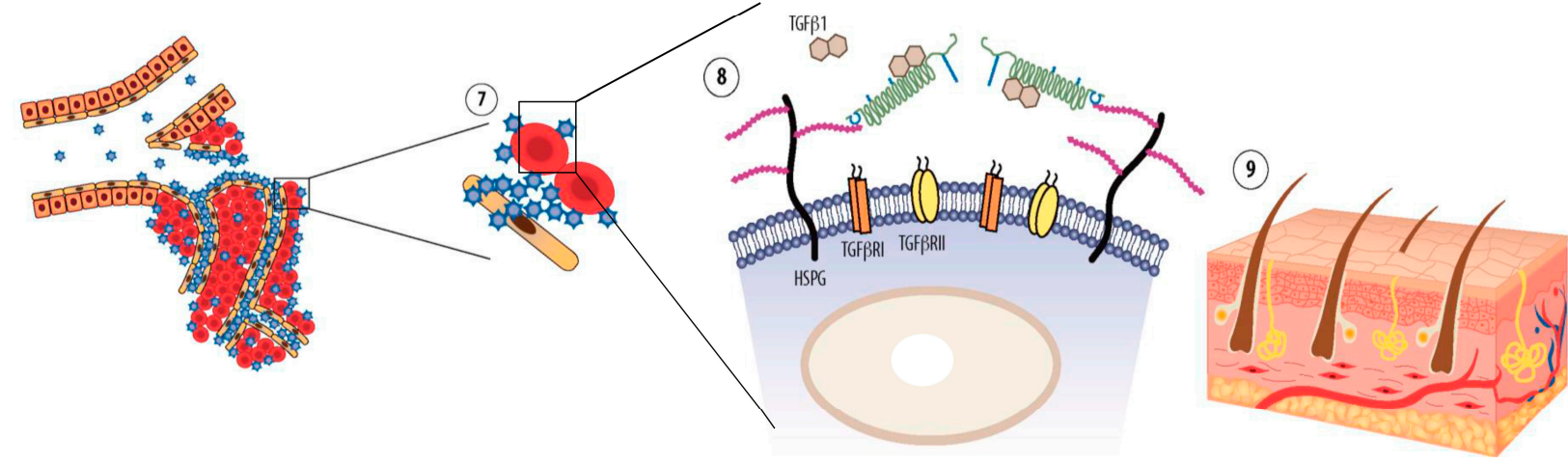

Figure 5. Schematic representation of the mechanism of action of the systemically administered, target organ-specific anti-fibrotic molecule, CAR-decorin. CAR-decorin (1) is a systemically administered, target-seeking, multi-functional biotherapeutic that inhibits scar formation. The molecule can be targeted to injury taking place at any organ of the body $(2,3)$ (or multiple organs simultaneously). The CAR homing peptide targets angiogenic vasculature, which forms at the site of the injury $(4,5)$. The peptide (and any payload attached to it; blue stars) then extravasates into surrounding tissue (6), where it binds to its receptor(s) on the cell surface of the scar producing fibroblasts (7). CAR binding to heparan sulfate proteoglycans (HSPGs) provides docking sites in the proximity of the main scar-inducing growth factors TGF- $\beta 1$ and TGF- $\beta 2(8)$ facilitating the neutralization of these growth factors by the therapeutic part of the molecule, decorin (8). This mechanism results in therapeutic response (9) seen as reduced scar formation in the skin [39]. Picture by Helena Schmidt; reproduced after modification with permission from [57], Copyright 2011 Finnish Medical Journal Duodecim. 
A

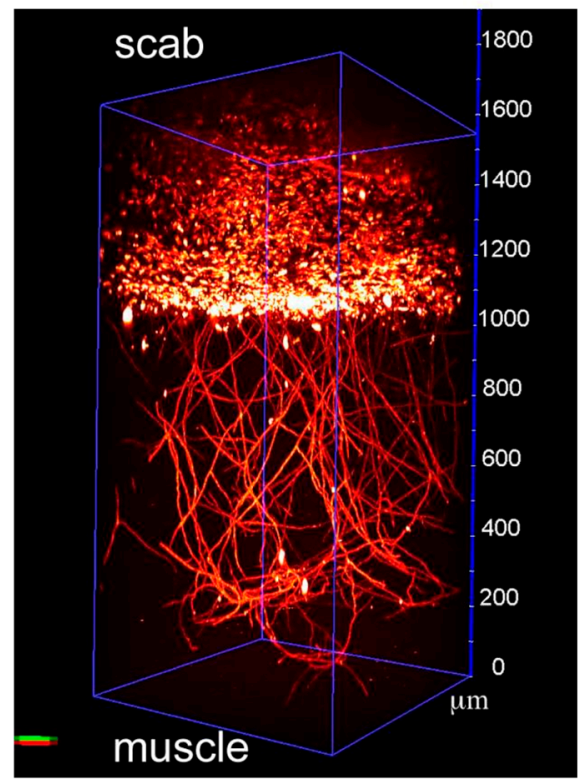

B

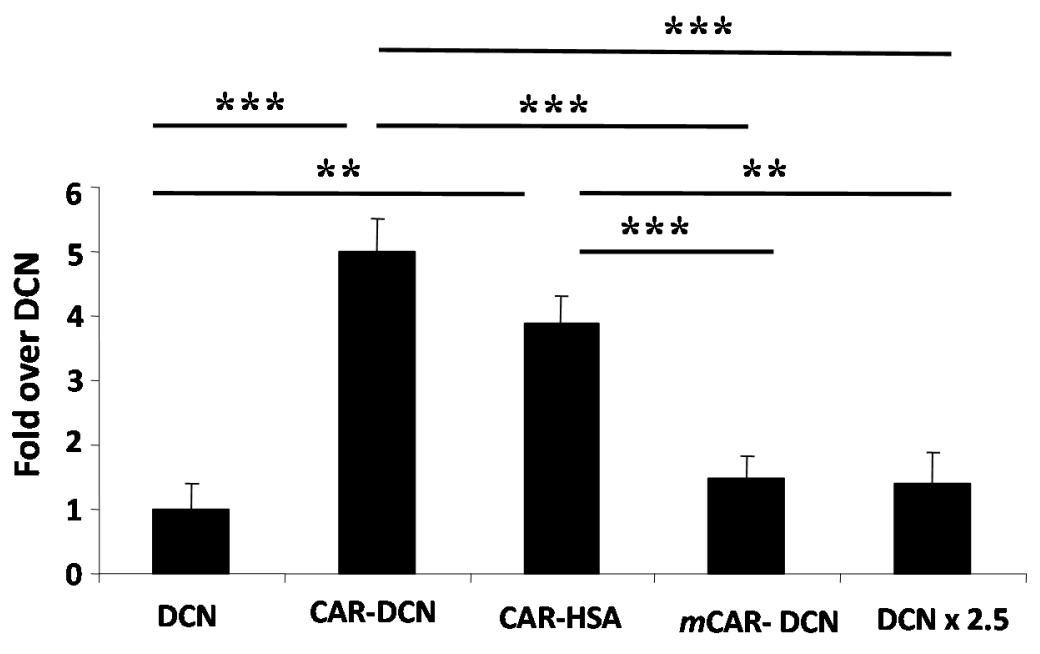

Figure 6. Targeted delivery of systemically administered therapeutics to the regenerating tissues. (A) 2-Photon fluorescence microscopy of vascular homing peptide accumulation to wound vasculature illustrates the specificity of the vascular targeting [30]. 2-Photon fluorescence microscope allows 3-D imaging of whole tissues [30]; and (B) Mice with full thickness skin wounds received an i.v. injection of His-tagged fusion proteins. The location of decorin was determined with an anti-His-tag antibody. The wounds of mice injected with non-targeted decorin (DCN) or mutant CAR peptide coupled decorin ( $m$ CAR-DCN) were weakly positive, whereas strong wound staining was observed in CAR-DCN injected mice [39]. The accumulation of CAR fused to human serum albumin (CAR-HSA) in the wounds confirmed the ability of the CAR peptide to enhance targeting to the wounds in fusion proteins other than DCN [39]. No staining was observed in normal skeletal muscle underlying the skin wounds of mice treated with any of the decorins. The staining was quantified by digital histology. The statistical significance was examined with ANOVA; $* * p \leq 0.01, * * * p<0.001[39]$.

\section{Future Perspectives}

In diseases affecting lungs, the CAR peptide has also recently been shown to target inflammatory vasculature (Figure 4) [42,47,57-61] and to deliver different pharmaceutical agents in a target organ-specific fashion to diseased lungs (Figure 4) [42]. Furthermore, both CAR and CRK peptides have been used to target mesenchymal stem cells to infarcted myocardium [38]. Thus, CAR-targeted therapeutic molecules could also be useful in the treatment of chronic, inflammatory diseases (outside of healing wounds), or in diseases where there is angiogenesis or inflammation affecting the local vasculature. 


\section{Acknowledgments}

The work was funded by the Sigrid Juselius Foundation, the Academy of Finland, Päivikki and Sakari Sohlberg Foundation, Instrumentarium Research Foundation, Tampere Tuberculosis Foundation, Finnish Medical Foundation, Pirkanmaa Hospital District Research Foundation and the Finnish Cultural Foundation.

\section{Author Contributions}

Tero A. H. Järvinen, Ulrike May and Stuart Prince wrote the manuscript.

\section{Conflicts of Interest}

The authors declare no conflict of interest.

\section{References}

1. Eming, S.A.; Martin, P.; Tomic-Canic, M. Wound repair and regeneration: Mechanisms, signaling, and translation. Sci. Transl. Med. 2014, 6, $265 \mathrm{sr} 6$.

2. Järvinen, T.A.; Järvinen, T.L.; Kääriäinen, M.; Kalimo, H.; Järvinen, M. Muscle injuries: Biology and treatment. Am. J. Sports Med. 2005, 33, 745-764.

3. Gurtner, G.C.; Werner, S.; Barrandon, Y.; Longaker, M.T. Wound repair and regeneration. Nature 2008, 453, 314-321.

4. Martino, M.M.; Briquez, P.S.; Maruyama, K.; Hubbell, J.A. Extracellular matrix-inspired growth factor delivery systems for bone regeneration. Adv. Drug Deliv. Rev. 2015, doi:10.1016/j.addr.2015.04.007.

5. Geer, D.J.; Swartz, D.D.; Andreadis, S.T. Biomimetic delivery of keratinocyte growth factor upon cellular demand for accelerated wound healing in vitro and in vivo. Am. J. Pathol. 2005, 167, 1575-1586.

6. Martino, M.M.; Briquez, P.S.; Guc, E.; Tortelli, F.; Kilarski, W.W.; Metzger, S.; Rice, J.J.; Kuhn, G.A.; Müller, R.; Swartz, M.A.; et al. Growth factors engineered for super-affinity to the extracellular matrix enhance tissue healing. Science 2014, 343, 885-888.

7. Arap, W.; Pasqualini, R.; Ruoslahti, E. Cancer treatment by targeted drug delivery to tumor vasculature in a mouse model. Science 1998, 279, 377-380.

8. Hussain, S.; Rodriguez-Fernandez, M.; Braun, G.B.; Doyle, F.J.; Ruoslahti, E. Quantity and accessibility for specific targeting of receptors in tumours. Sci. Rep. 2014, 4, 5232.

9. Ruoslahti, E.; Rajotte, D. An address system in the vasculature of normal tissues and tumors. Annu. Rev. Immunol. 2000, 18, 813-827.

10. AlDeghaither, D.; Smaglo, B.G.; Weiner, L.M. Beyond peptides and mAbs - Current status and future perspectives for biotherapeutics with novel constructs. J. Clin. Pharmacol. 2015, 55 (Suppl. 3), S4-S20.

11. Ruoslahti, E.; Bhatia, S.N.; Sailor, M.J. Targeting of drugs and nanoparticles to tumors. J. Cell Biol. 2010, 188, 759-768.

12. Teesalu, T.; Sugahara, K.N.; Ruoslahti, E. Tumor-penetrating peptides. Front. Oncol. 2013, 3, 216. 
13. Huang, R.; Ma, H.; Guo, Y.; Liu, S.; Kuang, Y.; Shao, K.; Li, J.; Liu, Y.; Han, L.; Huang, S.; et al. Angiopep-conjugated nanoparticles for targeted long-term gene therapy of Parkinson's disease. Pharm. Res. 2013, 30, 2549-2559.

14. Pasqualini, R.; Ruoslahti, E. Organ targeting in vivo using phage display peptide libraries. Nature 1996, 380, 364-366.

15. Nolan, D.J.; Ginsberg, M.; Israely, E.; Palikuqi, B.; Poulos, M.G.; James, D.; Ding, B.-S.; Schachterle, W.; Liu, Y.; Rosenwaks, Z.; et al. Molecular signatures of tissue-specific microvascular endothelial cell heterogeneity in organ maintenance and regeneration. Dev. Cell 2013, 26, 204-219.

16. Oh, P.; Borgstrom, P.; Witkiewicz, H.; Li, Y.; Borgstrom, B.J.; Chrastina, A.; Iwata, K.; Zinn, K.R.; Baldwin, R.; Testa, J.E.; et al. Live dynamic imaging of caveolae pumping targeted antibody rapidly and specifically across endothelium in the lung. Nat. Biotechnol. 2007, 25, 327-337.

17. Oh, P.; Testa, J.E.; Borgstrom, P.; Witkiewicz, H.; Li, Y.; Schnitzer, J.E. In vivo proteomic imaging analysis of caveolae reveals pumping system to penetrate solid tumors. Nat. Med. 2014, 20, $1062-1068$.

18. Rajotte, D.; Arap, W.; Hagedorn, M.; Koivunen, E.; Pasqualini, R.; Ruoslahti, E. Molecular heterogeneity of the vascular endothelium revealed by in vivo phage display. J. Clin. Investig. 1998, 102, 430-437.

19. Ruoslahti, E. Vascular zip codes in angiogenesis and metastasis. Biochem. Soc. Trans. 2004, 32, 397-402.

20. Järvinen, T.A.; Ruoslahti, E. Molecular changes in the vasculature of injured tissues. Am. J. Pathol. 2007, 171, 702-711.

21. De Francesco, F.; Tirino, V.; Desiderio, V.; Ferraro, G.; D’Andrea, F.; Giuliano, M.; Libondi, G.; Pirozzi, G.; de Rosa, A.; Papaccio, G. Human CD34/CD90 ASCs are capable of growing as sphere clusters, producing high levels of VEGF and forming capillaries. PLoS ONE 2009, 4, e6537.

22. Teesalu, T.; Sugahara, K.N.; Ruoslahti, E. Mapping of vascular ZIP codes by phage display. Methods Enzymol. 2012, 503, 35-56.

23. Järvinen, T.A. Design of target-seeking antifibrotic compounds. Methods Enzymol. 2012, 509, 243-261.

24. Zhang, L.; Hoffman, J.A.; Ruoslahti, E. Molecular profiling of heart endothelial cells. Circulation 2005, 112, 1601-1611.

25. Whitney, M.A.; Crisp, J.L.; Nguyen, L.T.; Friedman, B.; Gross, L.A.; Steinbach, P.; Tsien, R.Y.; Nguyen, Q.T. Fluorescent peptides highlight peripheral nerves during surgery in mice. Nat. Biotechnol. 2011, 29, 352-356.

26. Schafer, M.; Werner, S. Cancer as an overhealing wound: An old hypothesis revisited. Nat. Rev. Mol. Cell Biol. 2008, 9, 628-638.

27. Papaccio, G. Insulitis and islet microvasculature in type 1 diabetes. Histol. Histopathol. 1993, 8, 751-759.

28. Järvinen, M. Healing of a crush injury in rat striated muscle. 3. A micro-angiographical study of the effect of early mobilization and immobilization on capillary ingrowth. Acta Pathol. Microbiol. Scand. Sect. A Pathol. 1976, 84, 85-94.

29. Järvinen, T.A.; Ruoslahti, E. Targeted antiscarring therapy for tissue injuries. Adv. Wound Care 2013, 2, 50-54. 
30. Yanez, C.O.; Morales, A.R.; Yue, X.; Urakami, T.; Komatsu, M.; Järvinen, T.A.; Belfield, K.D. Deep vascular imaging in wounds by two-photon fluorescence microscopy. PLoS ONE 2013, 8, e67559.

31. Agemy, L.; Sugahara, K.N.; Kotamraju, V.R.; Gujraty, K.; Girard, O.M.; Kono, Y.; Mattrey, R.F.; Park, J.-H.; Sailor, M.J.; Jimenez, A.I.; et al. Nanoparticle-induced vascular blockade in human prostate cancer. Blood 2010, 116, 2847-2856.

32. Doll, F.; Schwager, K.; Hemmerle, T.; Neri, D. Murine analogues of etanercept and of F8-IL10 inhibit the progression of collagen-induced arthritis in the mouse. Arthritis Res. Ther. 2013, 15, R138.

33. Franz, M.; Doll, F.; Grun, K.; Richter, P.; Kose, N.; Ziffels, B.; Schubert, H.; Figulla, H.R.; Jung, C.; Gummert, J.; et al. Targeted delivery of interleukin-10 to chronic cardiac allograft rejection using a human antibody specific to the extra domain A of fibronectin. Int. J. Cardiol. 2015, 195, 311-322.

34. Galeazzi, M.; Bazzichi, L.; Sebastiani, G.D.; Neri, D.; Garcia, E.; Ravenni, N.; Giovannoni, L.; Wilton, J.; Bardelli, M.; Baldi, C.; et al. A phase IB clinical trial with Dekavil (F8-IL10), an immunoregulatory "armed antibody" for the treatment of rheumatoid arthritis, used in combination wilh methotrexate. Isr. Med. Assoc. J. 2014, 16, 666.

35. Schwager, K.; Kaspar, M.; Bootz, F.; Marcolongo, R.; Paresce, E.; Neri, D.; Trachsel, E. Preclinical characterization of DEKAVIL (F8-IL10), a novel clinical-stage immunocytokine which inhibits the progression of collagen-induced arthritis. Arthritis Res. Ther. 2009, 11, R142.

36. Corti, A.; Curnis, F. Tumor vasculature targeting through NGR peptide-based drug delivery systems. Curr. Pharm. Biotechnol. 2011, 12, 1128-1134.

37. Calcinotto, A.; Grioni, M.; Jachetti, E.; Curnis, F.; Mondino, A.; Parmiani, G.; Corti, A.; Bellone, M. Targeting TNF- $\alpha$ to neoangiogenic vessels enhances lymphocyte infiltration in tumors and increases the therapeutic potential of immunotherapy. J. Immunol. 2012, 188, 2687-2694.

38. Kean, T.J.; Duesler, L.; Young, R.G.; Dadabayev, A.; Olenyik, A.; Penn, M.; Wagner, J.; Fink, D.J.; Caplan, A.I.; Dennis, J.E. Development of a peptide-targeted, myocardial ischemia-homing, mesenchymal stem cell. J. Drug Target. 2012, 20, 23-32.

39. Järvinen, T.A.; Ruoslahti, E. Target-seeking antifibrotic compound enhances wound healing and suppresses scar formation in mice. Proc. Natl. Acad. Sci. USA 2010, 107, 21671-21676.

40. Ma, W.; Cai, S.; Du, J.; Tan, Y.; Chen, H.; Guo, Z.; Hu, H.; Fang, R.; Cai, S. SDF-1/54-DCN: A novel recombinant chimera with dual inhibitory effects on proliferation and chemotaxis of tumor cells. Biol. Pharm. Bull. 2008, 31, 1086-1091.

41. Kean, T.J.; Lin, P.; Caplan, A.I.; Dennis, J.E. MSCs: Delivery routes and engraftment, cell-targeting strategies, and immune modulation. Stem Cells Int. 2013, $2013,732742$.

42. Toba, M.; Alzoubi, A.; O’Neill, K.; Abe, K.; Urakami, T.; Komatsu, M.; Alvarez, D.; Järvinen, T.A.H.; Mann, D.; Ruoslahti, E.; et al. A novel vascular homing peptide strategy to selectively enhance pulmonary drug efficacy in pulmonary arterial hypertension. Am. J. Pathol. 2014, 184, 369-375.

43. Sugahara, K.N.; Teesalu, T.; Karmali, P.P.; Kotamraju, V.R.; Agemy, L.; Girard, O.M.; Hanahan, D.; Mattrey, R.F.; Ruoslahti, E. Tissue-penetrating delivery of compounds and nanoparticles into tumors. Cancer Cell 2009, 16, 510-520. 
44. Teesalu, T.; Sugahara, K.N.; Kotamraju, V.R.; Ruoslahti, E. C-end rule peptides mediate neuropilin-1-dependent cell, vascular, and tissue penetration. Proc. Natl. Acad. Sci. USA 2009, 106, 16157-16162.

45. Sugahara, K.N.; Teesalu, T.; Karmali, P.P.; Kotamraju, V.R.; Agemy, L.; Greenwald, D.R.; Ruoslahti, E. Coadministration of a tumor-penetrating peptide enhances the efficacy of cancer drugs. Science 2010, 328, 1031-1035.

46. Ruoslahti, E. The RGD story: A personal account. Matrix Biol. 2003, 22, 459-465.

47. Urakami, T.; Jarvinen, T.A.; Toba, M.; Sawada, J.; Ambalavanan, N.; Mann, D.; McMurtry, I.; Oka, M.; Ruoslahti, E.; Komatsu, M. Peptide-directed highly selective targeting of pulmonary arterial hypertension. Am. J. Pathol. 2011, 178, 2489-2495.

48. Ruoslahti, E.; Yamaguchi, Y. Proteoglycans as modulators of growth factor activities. Cell 1991, 64, 867-869.

49. Border, W.A.; Ruoslahti, E. Transforming growth factor- $\beta$ in disease: The dark side of tissue repair. J. Clin. Investig. 1992, 90, 1-7.

50. Järvinen, T.A.H.; Prince, S. Decorin-A growth factor antagonist for tumor growth inhibition. BioMed Res. Int. 2015, in press.

51. Border, W.A.; Noble, N.A.; Yamamoto, T.; Harper, J.R.; Yamaguchi, Y.; Pierschbacher, M.D.; Ruoslahti, E. Natural inhibitor of transforming growth factor- $\beta$ protects against scarring in experimental kidney disease. Nature 1992, 360, 361-364.

52. Yamaguchi, Y.; Ruoslahti, E. Expression of human proteoglycan in Chinese hamster ovary cells inhibits cell proliferation. Nature 1988, 336, 244-246.

53. Yamaguchi, Y.; Mann, D.M.; Ruoslahti, E. Negative regulation of transforming growth factor- $\beta$ by the proteoglycan decorin. Nature 1990, 346, 281-284.

54. Zhu, J.; Li, Y.; Shen, W.; Qiao, C.; Ambrosio, F.; Lavasani, M.; Nozaki, M.; Branca, M.F.; Huard, J. Relationships between transforming growth factor- $\beta 1$, myostatin, and decorin: Implications for skeletal muscle fibrosis. J. Biol. Chem. 2007, 282, 25852-25863.

55. Vial, C.; Gutierrez, J.; Santander, C.; Cabrera, D.; Brandan, E. Decorin interacts with connective tissue growth factor (CTGF)/CCN2 by LRR12 inhibiting its biological activity. J. Biol. Chem. 2011, 286, 24242-24252.

56. Andrianifahanana, M.; Wilkes, M.C.; Gupta, S.K.; Rahimi, R.A.; Repellin, C.E.; Edens, M.; Wittenberger, J.; Yin, X.; Maidl, E.; Becker, J.; et al. Profibrotic TGF- $\beta$ responses require the cooperative action of PDGF and ErbB receptor tyrosine kinases. FASEB J. 2013, 27, 4444-4454.

57. Järvinen, T.A.H.; Ruoslahti, E. Uusi lääkeaine estää arven muodostusta. Duodecim 2011, 127, 146-147. (In Finnish)

58. Nahar, K.; Absar, S.; Gupta, N.; Kotamraju, V.R.; McMurtry, I.F.; Oka, M.; Komatsu, M.; Nozik-Grayck, E.; Ahsan, F. Peptide-coated liposomal fasudil enhances site specific vasodilation in pulmonary arterial hypertension. Mol. Pharm. 2014, 11, 4374-4384.

59. Gupta, N.; Patel, B.; Nahar, K.; Ahsan, F. Cell permeable peptide conjugated nanoerythrosomes of fasudil prolong pulmonary arterial vasodilation in PAH rats. Eur. J. Pharm. Biopharm. 2014, 88, $1046-1055$. 
60. Gupta, N.; Ibrahim, H.M.; Ahsan, F. Peptide-micelle hybrids containing fasudil for targeted delivery to the pulmonary arteries and arterioles to treat pulmonary arterial hypertension. J. Pharm. Sci. 2014, 103, 3743-3753.

61. Gupta, N.; Al-Saikhan, F.I.; Patel, B.; Rashid, J.; Ahsan, F. Fasudil and SOD packaged in peptide-studded-liposomes: Properties, pharmacokinetics and ex vivo targeting to isolated perfused rat lungs. Int. J. Pharm. 2015, 488, 33-43.

(C) 2015 by the authors; licensee MDPI, Basel, Switzerland. This article is an open access article distributed under the terms and conditions of the Creative Commons Attribution license (http://creativecommons.org/licenses/by/4.0/). 